\title{
Survival Benefit of Adjuvant Chemotherapy After Pancreatoduodenectomy for Ampullary Adenocarcinoma: a Propensity-Matched National Cancer Database (NCDB) Analysis
}

\author{
Sivesh K. Kamarajah ${ }^{1,2,3}$ - Filip Bednar ${ }^{4}$. Clifford S. Cho ${ }^{4} \cdot$ Hari Nathan ${ }^{4}$ \\ Received: 20 July 2020 / Accepted: 10 November 2020 / Published online: 23 November 2020 \\ (C) 2020 The Author(s)
}

\begin{abstract}
Background The benefit of adjuvant chemotherapy (AC) after pancreatoduodenectomy (PD) for ampullary adenocarcinoma is uncertain. We aimed to evaluate the association of $\mathrm{AC}$ with survival in patients with resected ampullary adenocarcinoma.

Methods Using the National Cancer Database (NCDB) data from 2004 to 2016, patients with non-metastatic ampullary adenocarcinoma who underwent PD were identified. Patients with neoadjuvant radiotherapy and chemotherapy and survival $<6$ months were excluded. Propensity score matching was used to account for treatment selection bias. A multivariable Cox proportional hazards model was then used to analyze the association of AC with survival.

Results Of 3186 (43\%) AC and 4172 (57\%) no AC (noAC) patients, $1720 \mathrm{AC}$ and 1720 noAC patients remained in the cohort after matching. Clinicopathologic variables were well balanced after matching. After matching, AC was associated with improved survival (median 47.5 vs 39.6 months, $p=0.003$ ), which remained after multivariable adjustment (HR: $0.83, \mathrm{CI}_{95 \%}: 0.76-$ $0.91, p<0.001$ ). Multivariable interaction analyses showed that this benefit was seen irrespective of nodal status: N0 (HR: 0.81 , $\mathrm{CI}_{95 \%}$ : 0.68-0.97, $p<0.001$ ), N1 (HR: 0.65, $\mathrm{CI}_{95 \%}: 0.61-0.70, p<0.001$ ), N2 (HR: 0.73, $\mathrm{CI}_{95 \%}$ : 0.59-0.90, $p=0.003$ ), N3 (HR: $0.59, \mathrm{CI}_{95 \%}: 0.44-0.78, p<0.001$ ); and margin status: R0 (HR: 0.85, CI $95 \%$ : 0.77-0.94, $p<0.001$ ), R1 (HR: 0.69, CI $95 \%$ : 0.48$1.00, p<0.001)$. Stratified analyses by nodal and margin status demonstrated consistent results.

Conclusion In this large retrospective cohort study, AC after resected ampullary adenocarcinoma was associated with a survival benefit in patients, including patients with node-negative and margin-negative disease.
\end{abstract}

Keywords Ampullary cancer · Adjuvant therapy $\cdot$ Resection

\section{Introduction}

Ampullary adenocarcinoma typically has a better long-term prognosis after curative resection than other periampullary cancers, with 5 -year survival rates ranging from 30 to

Hari Nathan

drnathan@umich.edu

1 Department of Surgery, University Hospital Birmingham NHS Trust, Birmingham, UK

2 Department of Hepatobiliary, Pancreatic and Transplant Surgery, Freeman Hospital, Newcastle Upon Tyne, Tyne and Wear, UK

3 Department of Surgery, Freeman Hospital, Newcastle Upon Tyne, Tyne and Wear, UK

4 Department of Surgery, 2210A Taubman Health Care Center, University of Michigan, 1500 E Medical Center Dr, SPC 5343, Ann Arbor, MI 48109-5343, USA
$70 \% .^{1-5}$ Despite this, up to $50 \%$ of patients have recurrence, ${ }^{6,7}$ with some series ${ }^{8,9}$ demonstrating similar rates of locoregional and distant recurrence while others ${ }^{10,11}$ suggesting predominance of distant recurrence. Adjuvant chemotherapy (AC) may help reduce both locoregional and distant recurrence rates and improve overall survival. While multiple randomized controlled trials have conclusively established the survival benefit of AC for pancreatic cancer, ${ }^{12-17}$ its role is not yet clear for ampullary adenocarcinoma.

High-quality evidence on AC for periampullary adenocarcinoma is lacking. First, randomized controlled trials (RCTs) ${ }^{18-21}$ and meta-analyses ${ }^{22,23}$ have demonstrated no survival benefit. However, different periampullary cancers (i.e., distal cholangiocarcinoma, duodenal adenocarcinoma, ampullary adenocarcinoma, and pancreatic adenocarcinoma) have varying prognoses, genetic profiles ${ }^{24}$, and likely responses to AC. ${ }^{25}$ Because ampullary adenocarcinoma is relatively uncommon, recruitment to RCTs has only been possible together with other 
periampullary cancer, and no RCT focused on ampullary adenocarcinoma exists. Subgroup analyses of these RCTs have limited interpretability and are prone to type II error. Retrospective single-center, multi-institutional series offer conflicting evidence regarding the benefit of AC. ${ }^{2,10,25-31}$ Therefore, the use of AC after pancreatoduodenectomy (PD) for ampullary adenocarcinoma remains controversial, especially in patients thought to be at a lower risk for recurrence, such as those with margin-negative resections and node-negative disease.

We sought to add evidence to this debate by performing a large, nationwide, high-quality retrospective study to assess the potential benefit of AC after PD for ampullary adenocarcinoma. With contemporary data from the National Cancer Data Base (NCDB), the association of AC with survival after PD for ampullary adenocarcinoma was analyzed. Propensitymatched analysis was used to address treatment selection bias, and overall survival in clinically relevant subgroups of patients based on nodal and margin status was assessed.

\section{Methods}

\section{Data Source}

The NCDB is a joint project of the Commission on Cancer $(\mathrm{CoC})$ of the American College of Surgeons and the American Cancer Society. ${ }^{32,33}$ The NCDB gathers information from approximately $1500 \mathrm{CoC}$-accredited hospitals and includes > $70 \%$ of all newly diagnosed malignancies in the USA. It contains specific details about patient demographics (age, sex, race, payer), facility type and location, tumor characteristics (size, grade, stage, histology), treatment course (type of surgery, receipt of chemotherapy, and radiation therapy), and outcomes (resection margins, lymph node status, and vital status).

\section{Study Population}

The NCDB was used to identify all patients $>35$ years old diagnosed with non-metastatic ampullary adenocarcinoma undergoing PD between 2004 and 2016. The International Classification of Disease for Oncology, Third Edition (ICDO-3), classification was used to select adenocarcinoma histology (8140-8148) and excluded mucinous tumors, neuroendocrine tumors, and other histologies. Patients with other concomitant cancer diagnoses, those who received neoadjuvant chemotherapy or radiotherapy, and those with missing data on lymph node status and survival $<6$ months were excluded.

The following patient-level characteristics were analyzed as provided by NCDB: age $(36-50,51-65,66-80,>80)$, race (white, black, other), Charlson-Deyo comorbidity score (CDCC), year of diagnosis, insurance status (Medicaid/ Medicare, private insurance, uninsured), zip code-level education status $(<7 \%, 7-12.9 \%, 13-20.9 \%, \geq 21 \%)$, zip code-level median household income $(<\$ 48,000, \$ 48,000-\$ 62,999, \geq$ $\$ 63,000)$, and urban versus rural area of residence. The zip code-level education status represents the proportion of adults in the patient's zip code who did not graduate from high school and is categorized as equally proportioned quartiles among all US zip codes. The following hospital-level characteristics were also analyzed: facility type (academic, community, other), facility location (Midwest, Northeast, South, West), and hospital distance from patient ( $<12.5$ miles, $12.5-49.9$ miles, $\geq 50$ miles). Finally, the following clinicopathologic characteristics were analyzed: nodal status (N0, N1, N2, N3), tumor grade/ differentiation (well/moderate, poor/anaplastic, unknown), lymphovascular invasion (absent, present), and margin status (R0: negative; R1: positive).

Finally, the receipt of AC versus no adjuvant chemotherapy (noAC) as the primary exposure variable was analyzed. Coding for adjuvant therapy was derived using start of adjuvant therapy from diagnosis and surgery to obtain reliable estimates. However, discrimination between adjuvant radiotherapy-sensitizing chemotherapy was not possible based on the current available data.

\section{Statistical Analysis}

Categorical variables were compared using the chi-square test. Non-normally distributed data were analyzed using the MannWhitney $U$ test. Landmark analysis was also performed excluding early postoperative mortality (i.e., $<6$ months) to account for immortal time bias. ${ }^{34}$ Survival was estimated using Kaplan-Meier survival curves and compared using the log-rank test. Multivariable analyses used Cox proportional hazards models. The conditional probability of receiving AC (i.e., the propensity score) was estimated using a multivariable logistic regression model including all patient- and hospitallevel variables listed above. Next, balanced cohorts were created using 1-to-1 nearest-neighbor propensity score matching (PSM) without replacement (caliper width 0.1 standard deviations). ${ }^{35}$ Balance diagnostics were conducted by using standardized mean differences, with a value $<0.1$ indicating good balance. ${ }^{35}$ The overall survival (OS) of matched patients with and without adjuvant chemotherapy was then evaluated. In order to address any residual confounding after PSM, multivariable Cox proportional hazards models again adjusted for all variables listed above, in addition to PSM. A stratified survival analysis by pathological node status (N0, N1, N2, and N3) and margin status (R0, R1) and interaction analyses between $\mathrm{AC}$ and pathological nodal and margin status were performed. A $p$ value of $<0.05$ was considered to be statistically significant. Data analysis was performed using the $\mathrm{R}$ Foundation Statistical software (R 3.2.2) with TableOne, ggplot2, Hmisc, Matchit, and survival packages (R Foundation for Statistical Computing, Vienna, Austria) as previously described. ${ }^{36}$ The study was deemed exempt from 
review by the University of Michigan Institutional Review Board.

\section{Results}

\section{Patient Demographics and Clinicopathologic Characteristics}

This study included 7358 patients with resected ampullary adenocarcinoma. Of these patients, $3186(43 \%)$ received AC and 4172 (57\%) did not. Median follow-up was 28 months (interquartile range 13-54 months). Baseline demographics of the unmatched cohort revealed that patients receiving $\mathrm{AC}$ were from high hospital volume and younger and had lower comorbidity burden (Table 1). There was a wide variation in receipt of AC by institution ranging from 0 to $100 \%$ (Supplementary Figure 1). Patients receiving $\mathrm{AC}$ also had larger, more locally invasive tumors and more positive lymph nodes, consistent with treatment selection bias. Patients receiving AC had significantly higher rates of lymph nodes examined compared to noAC (median: 18 vs $14, p<0.001$ ). Patients with node-positive disease were much more likely to receive $\mathrm{AC}$ than those with node-negative disease ( $72 \%$ vs $40 \%, p<0.001)$. Patients with margin-positive disease were much more likely to receive $\mathrm{AC}$ than those with margin-negative disease (7\% vs $4 \%, p<0.001$ ). Logistic regression identified advanced tumor, nodal involvement, and lymphovascular invasion as independent predictors of receipt of chemotherapy (Supplementary Table 1). To account for this treatment selection bias, PSM was performed as described above. This resulted in well-balanced cohorts (Table 1). Standardized mean differences were calculated for each variable and ranged between 0.01 and 0.05 , indicating good balance.

\section{Association of Adjuvant Chemotherapy with Survival}

For the overall cohort, median survival was 40.2 months, and 5 -year survival was $40 \%$. In the unmatched cohort, the survival of patients receiving $\mathrm{AC}$ was significantly shorter than those who did not (median: 43.3 vs 50.2 months, 5 -year $42 \%$ vs $46 \%, p=0.013$ ) (Fig. 1a, Table 2, and Supplementary Table 2). In the matched cohort, patients receiving AC still had a significant survival advantage (median 47.5 vs 39.6 months, 5 -year $44 \%$ vs $40 \%, p<0.001$ ) (Fig. $1 \mathrm{~b}$ and Table 2). In the PSM multivariable analysis, factors associated with adverse survival included older age, higher comorbidity score, advanced tumors, node-positive tumors, positive margin status, and lymphovascular invasion (Table 3). Patients receiving AC had improved survival after PSM and multivariable adjustment (HR: $0.83, \mathrm{CI}_{95 \%}$ : $0.76-0.91, p<0.001$ ) (Tables 2 and 3 ).

\section{Interaction Between Adjuvant Chemotherapy and Nodal Status}

Interaction analyses were performed to further understand the impact of AC by nodal status. In unadjusted analysis, there were significant differences in survival between $\mathrm{AC}$ and noAC patients in patients with $\mathrm{N} 1$ disease (median 39.1 vs 34.4 months, $p=0.014$ ) (Fig. 2a) and N3 disease (median 26.1 vs 21.0 months, $p=0.011$ ) (Fig. 2b) but not N0 disease (median 90.0 vs 86.1 months, $p=0.1$ ) (Supplementary Figure 2A) and N2 disease (median 27.3 vs 23.8 months, $p=0.5$ ) (Supplementary Figure 2B). In multivariable analyses modeling the interaction between receipt of $\mathrm{AC}$ and nodal status, a survival benefit again was seen for patients with N0, N1, N2, and N3 disease (Table 4 and Supplementary Table 3). As a sensitivity analysis, four separate multivariable analyses in cohorts including only those with $\mathrm{N} 0, \mathrm{~N} 1, \mathrm{~N} 2$, and $\mathrm{N} 3$ disease were performed, respectively. These analyses confirmed the same findings (Table 2).

\section{Interaction Between Adjuvant Chemotherapy and Margin Status}

Interaction analyses were performed to further understand the impact of AC by margin status. In unadjusted analysis, there were significant differences in survival between $\mathrm{AC}$ and noAC patients in patients with R0 disease (median 49.2 vs 42.3 months, $p<0.001$ ) (Fig. 3a) and in patients with R1 disease (median 22.3 vs 17.5 months, $p=0.016$ ) (Fig. 3b). In multivariable analyses modeling the interaction between receipt of $\mathrm{AC}$ and margin status, a survival benefit again was seen for patients with R0 (HR: $0.85, \mathrm{CI}_{95 \%}$ : 0.77-0.94, $p<0.001$ ) and $\mathrm{R} 1$ margin status (HR: $0.69, \mathrm{CI}_{95 \%}: 0.48-1.00$, $p<0.001$ ) (Table 4 and Supplementary Table 4). As a sensitivity analysis, we performed two separate multivariable analyses in cohorts including only those with R0 or R1 margin, respectively. These analyses confirmed the same findings (Table 2).

\section{Association of Adjuvant Chemotherapy and Radiotherapy with Survival}

Additional analyses were performed to further understand the impact of AC in the setting of adjuvant radiotherapy. In unadjusted analysis, there were no significant differences in survival between $\mathrm{AC}$ and noAC patients in patients without adjuvant radiotherapy (median 44.8 vs 42.0 months, $p=0.2$ ) (Supplementary Figure 3A), but significantly longer with $\mathrm{AC}$ than noAC in patients with adjuvant radiotherapy (median 51.1 vs 36.8 months, $p<0.001$ ) (Supplementary Figure 3B). In multivariable analyses modeling the interaction between receipt of AC and radiotherapy, a survival benefit again was seen for patients without adjuvant radiotherapy (HR: 0.81, 
Table 1 Clinicopathologic characteristics of ampullary adenocarcinoma by receipt of adjuvant chemotherapy in unmatched and matched cohort

\begin{tabular}{|c|c|c|c|c|c|c|c|}
\hline & & \multicolumn{3}{|c|}{ Unmatched cohort } & \multicolumn{3}{|c|}{ Matched cohort } \\
\hline & & $\begin{array}{l}\text { noAC } \\
n=4172\end{array}$ & $\begin{array}{l}\mathrm{AC} \\
n=3186\end{array}$ & $p$ value & $\begin{array}{l}\text { noAC } \\
n=1720\end{array}$ & $\begin{array}{l}\mathrm{AC} \\
n=1720\end{array}$ & $p$ value \\
\hline \multicolumn{8}{|l|}{ Hospital factors } \\
\hline \multirow{5}{*}{ Center volume } & 1 (lowest) & $508(12.2)$ & $573(18.0)$ & \multirow{5}{*}{$<0.001$} & $261(15.2)$ & $261(15.2)$ & \multirow{5}{*}{0.998} \\
\hline & 2 & $774(18.6)$ & $648(20.3)$ & & $325(18.9)$ & $327(19.0)$ & \\
\hline & 3 & $854(20.5)$ & $601(18.9)$ & & $328(19.1)$ & $328(19.1)$ & \\
\hline & 4 & $968(23.2)$ & $673(21.1)$ & & $383(22.3)$ & $389(22.6)$ & \\
\hline & 5 (highest) & 1068 (25.6) & $691(21.7)$ & & $423(24.6)$ & $415(24.1)$ & \\
\hline \multirow[t]{3}{*}{ Facility type } & Community & $1208(29.0)$ & $1038(32.6)$ & \multirow[t]{3}{*}{$<0.001$} & $954(55.5)$ & $953(55.4)$ & \multirow[t]{3}{*}{0.933} \\
\hline & Academic & 2451 (58.7) & $1665(52.3)$ & & $535(31.1)$ & $529(30.8)$ & \\
\hline & Others & $513(12.3)$ & $483(15.2)$ & & $231(13.4)$ & $238(13.8)$ & \\
\hline \multirow[t]{4}{*}{ Facility location } & Northeast & $889(21.3)$ & 714 (22.4) & \multirow[t]{4}{*}{0.166} & 409 (23.8) & $411(23.9)$ & \multirow[t]{4}{*}{0.479} \\
\hline & South & $1580(37.9)$ & $1150(36.1)$ & & $358(20.8)$ & $390(22.7)$ & \\
\hline & Midwest & $1031(24.7)$ & $764(24.0)$ & & $646(37.6)$ & $609(35.4)$ & \\
\hline & West & $672(16.1)$ & $558(17.5)$ & & $307(17.8)$ & $310(18.0)$ & \\
\hline \multicolumn{8}{|l|}{ Patient factors } \\
\hline \multirow{5}{*}{ Year of diagnosis } & 2006-2007 & $1646(39.5)$ & $431(13.5)$ & \multirow{5}{*}{$<0.001$} & 385 (22.4) & $375(21.8)$ & 0.339 \\
\hline & 2008-2009 & 585 (14.0) & $545(17.1)$ & & $249(14.5)$ & 235 (13.7) & \\
\hline & 2010-2011 & $647(15.5)$ & 608 (19.1) & & $295(17.2)$ & 269 (15.6) & \\
\hline & 2012-2013 & $696(16.7)$ & $766(24.0)$ & & $402(23.4)$ & $404(23.5)$ & \\
\hline & 2014-2016 & $598(14.3)$ & $836(26.2)$ & & 389 (22.6) & $437(25.4)$ & \\
\hline Age at diagnosis (years) & $36-50$ & $272(6.5)$ & $384(12.1)$ & $<0.001$ & $156(9.1)$ & $179(10.4)$ & 0.420 \\
\hline & $51-65$ & $1339(32.1)$ & $1374(43.1)$ & & $661(38.4)$ & $672(39.1)$ & \\
\hline & $66-80$ & 1998 (47.9) & $1288(40.4)$ & & 789 (45.9) & $751(43.7)$ & \\
\hline & $\geq 80$ & $560(13.4)$ & $134(4.2)$ & & $112(6.5)$ & $113(6.6)$ & \\
\hline Sex & Male & 2334 (55.9) & $1840(57.8)$ & 0.127 & $749(43.5)$ & $729(42.4)$ & 0.513 \\
\hline & Female & $1838(44.1)$ & $1346(42.2)$ & & $971(56.5)$ & $991(57.6)$ & \\
\hline CDCC score & $0-1$ & 3901 (93.5) & 3010 (94.5) & 0.093 & $1617(94.0)$ & 1619 (94.1) & 0.942 \\
\hline & $\geq 2$ & $271(6.5)$ & $176(5.5)$ & & $103(6.0)$ & $101(5.9)$ & \\
\hline Insurance status & Uninsured & $266(6.4)$ & $188(5.9)$ & $<0.001$ & $92(5.3)$ & $102(5.9)$ & 0.500 \\
\hline & Private insurance & $1323(31.7)$ & $1400(43.9)$ & & $856(49.8)$ & $827(48.1)$ & \\
\hline & Medicaid & $198(4.7)$ & $199(6.2)$ & & $652(37.9)$ & $683(39.7)$ & \\
\hline & Medicare & $2385(57.2)$ & $1399(43.9)$ & & $120(7.0)$ & $108(6.3)$ & \\
\hline Education level & $\geq 21 \%$ & $782(18.7)$ & $535(16.8)$ & $<0.001$ & $433(25.2)$ & $426(24.8)$ & 0.976 \\
\hline & $13-20.9 \%$ & $1085(26.0)$ & 738 (23.2) & & 299 (17.4) & 299 (17.4) & \\
\hline & $7-12.9 \%$ & $1337(32.0)$ & $1074(33.7)$ & & $415(24.1)$ & $410(23.8)$ & \\
\hline & $<7 \%$ & $968(23.2)$ & $839(26.3)$ & & $573(33.3)$ & $585(34.0)$ & \\
\hline Median income & $\leq \$ 47,999$ & 1738 (41.7) & $1141(35.8)$ & $<0.001$ & $636(37.0)$ & $622(36.2)$ & 0.808 \\
\hline & $\$ 48,000-\$ 62,999$ & $1128(27.0)$ & $902(28.3)$ & & $470(27.3)$ & $486(28.3)$ & \\
\hline & $\geq \$ 63,000$ & 1306 (31.3) & $1143(35.9)$ & & $614(35.7)$ & $612(35.6)$ & \\
\hline Tumor factors & & & & & & & \\
\hline Tumor grade & Well & $566(13.6)$ & $266(8.3)$ & $<0.001$ & $88(5.1)$ & $87(5.1)$ & 0.696 \\
\hline & Moderate & $2232(53.5)$ & $1632(51.2)$ & & $897(52.2)$ & $872(50.7)$ & \\
\hline & Poor & $1125(27.0)$ & $1125(35.3)$ & & $568(33.0)$ & $601(34.9)$ & \\
\hline & Anaplastic & $249(6.0)$ & $163(5.1)$ & & $167(9.7)$ & $160(9.3)$ & \\
\hline AJCC pathological T classification & $\mathrm{T} 1$ & $854(20.5)$ & $193(6.1)$ & $<0.001$ & $153(8.9)$ & $151(8.8)$ & 0.810 \\
\hline & $\mathrm{T} 2$ & $1400(33.6)$ & $876(27.5)$ & & $534(31.0)$ & $509(29.6)$ & \\
\hline & $\mathrm{T} 3$ & $1132(27.1)$ & $1178(37.0)$ & & $580(33.7)$ & $594(34.5)$ & \\
\hline & $\mathrm{T} 4$ & $786(18.8)$ & 939 (29.5) & & $453(26.3)$ & $466(27.1)$ & \\
\hline AJCC pathological $\mathrm{N}$ classification & No & $2574(61.7)$ & $896(28.1)$ & $<0.001$ & $679(39.5)$ & $644(37.4)$ & 0.389 \\
\hline & N1 & $1117(26.8)$ & $1469(46.1)$ & & $697(40.5)$ & $695(40.4)$ & \\
\hline & $\mathrm{N} 2$ & $310(7.4)$ & $491(15.4)$ & & $224(13.0)$ & $242(14.1)$ & \\
\hline & N3 & $171(4.1)$ & 330 (10.4) & & $120(7.0)$ & $139(8.1)$ & \\
\hline Margin status & Negative & $4021(96.4)$ & $2968(93.2)$ & $<0.001$ & $1640(95.3)$ & $1628(94.7)$ & 0.389 \\
\hline & Positive & $151(3.6)$ & $218(6.8)$ & & $80(4.7)$ & $92(5.3)$ & \\
\hline Lymphovascular invasion & Absent & $3584(85.9)$ & $2071(65.0)$ & $<0.001$ & $1257(73.1)$ & $1212(70.5)$ & 0.096 \\
\hline & Present & $588(14.1)$ & $1115(35.0)$ & & $463(26.9)$ & $508(29.5)$ & \\
\hline Treatment factors & & & & & & & \\
\hline Adjuvant radiotherapy & No & $3826(91.7)$ & $1609(50.5)$ & $<0.001$ & $1401(81.5)$ & 1396 (81.2) & 0.861 \\
\hline & Yes & $346(8.3)$ & $1577(49.5)$ & & $319(18.5)$ & $324(18.8)$ & \\
\hline
\end{tabular}

Additional variables included into the propensity matching omitted from tables were hospital factors (hospital distance), patient factors (race, residence), and tumor factors (lymph nodes examined)

$A C$ adjuvant chemotherapy, AJCC American Joint Commission on Cancer, $C D C C$ Charlson-Deyo comorbidity, noAC no adjuvant chemotherapy 

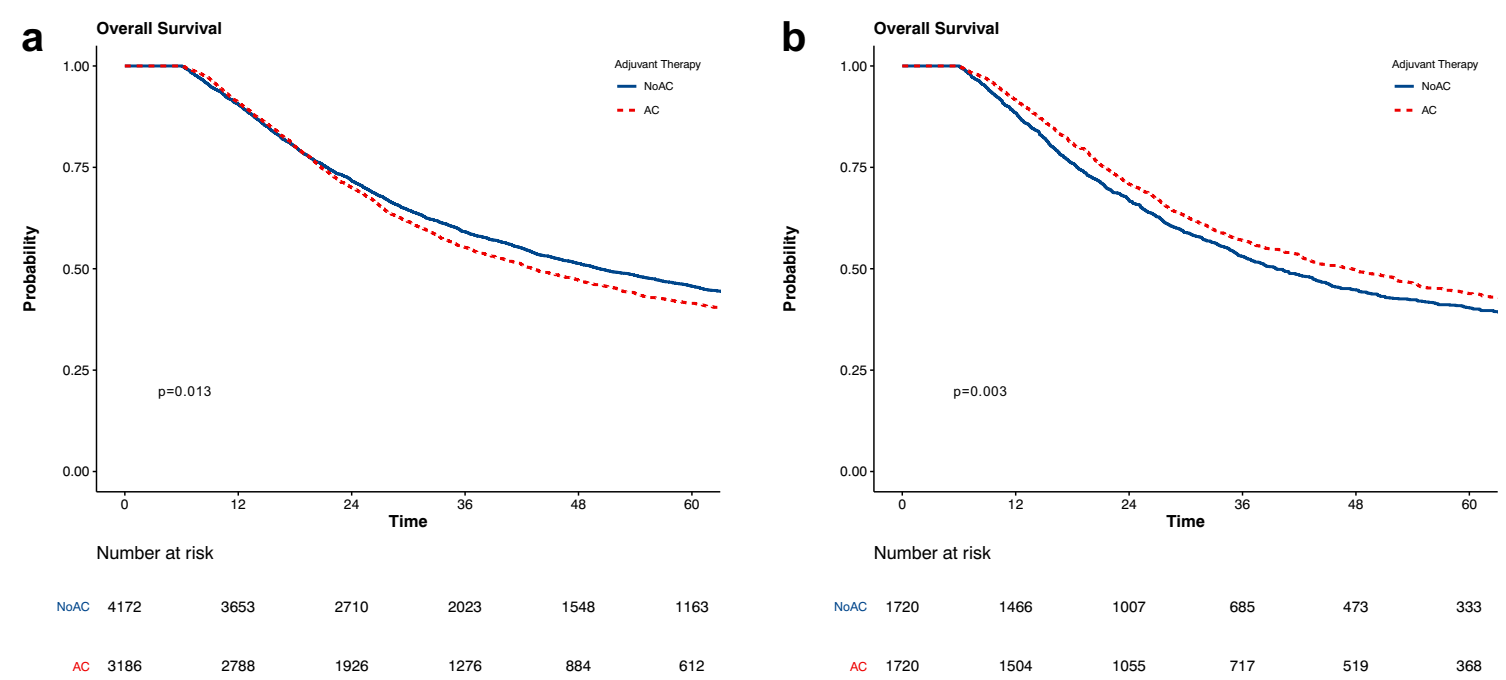

Fig. 1 Overall survival of adjuvant chemotherapy following resection for ampullary adenocarcinoma in $\mathbf{a}$ unmatched and $\mathbf{b}$ matched cohorts

$\left.\mathrm{CI}_{95 \%}: 0.68-0.96, p<0.001\right)$ and with adjuvant radiotherapy (HR: $0.56, \mathrm{CI}_{95 \%}$ : 0.47-0.66, $p<0.001$ ) (Supplementary Table 5). As a sensitivity analysis, we performed two separate multivariable analyses in cohorts including only those without and with adjuvant radiotherapy, respectively. These analyses confirmed the same findings (Table 2).
Table 2 Association of adjuvant chemotherapy with overall survival of patients with resected ampullary adenocarcinoma in unmatched and matched cohorts and stratified by nodal status and margin status for matched cohorts from multivariable Cox regression model

\begin{tabular}{|c|c|c|c|c|}
\hline Cohort & Chemotherapy & $\begin{array}{l}\text { Median survival (IQR), } \\
\text { months }\end{array}$ & $\begin{array}{l}\text { Hazard ratio } \\
\left(\mathrm{CI}_{95 \%}\right)\end{array}$ & $p$-value \\
\hline \multicolumn{5}{|l|}{ All patients } \\
\hline Unmatched & $\begin{array}{l}\text { noAC } \\
\mathrm{AC}\end{array}$ & $\begin{array}{l}50.2(47.5-54.1) \\
43.3(40.6-46.5)\end{array}$ & $\begin{array}{l}\text { REF } \\
0.85(0.78-0.92)\end{array}$ & $<0.001$ \\
\hline Matched & $\begin{array}{l}\text { noAC } \\
\mathrm{AC}\end{array}$ & $\begin{array}{l}39.6(36.6-43.7) \\
47.5(42.5-52.3)\end{array}$ & $\begin{array}{l}\text { REF } \\
0.83(0.76-0.91)\end{array}$ & $<0.001$ \\
\hline \multicolumn{5}{|c|}{ Stratified by nodal status in matched cohort } \\
\hline No & $\begin{array}{l}\text { noAC } \\
\mathrm{AC}\end{array}$ & $\begin{array}{l}86.1(78.5-104.0) \\
90.0(74.9-\mathrm{NR})\end{array}$ & $\begin{array}{l}\text { REF } \\
0.80(0.66-0.96)\end{array}$ & 0.017 \\
\hline N1 & $\begin{array}{l}\text { noAC } \\
\mathrm{AC}\end{array}$ & $\begin{array}{l}34.4(29.7-37.3) \\
39.1(34.4-44.8)\end{array}$ & $\begin{array}{l}\text { REF } \\
0.84(0.73-0.97)\end{array}$ & 0.014 \\
\hline $\mathrm{N} 2$ & $\begin{array}{l}\text { noAC } \\
\mathrm{AC}\end{array}$ & $\begin{array}{l}23.8(21.4-29.7) \\
27.3(23.9-30.3)\end{array}$ & $\begin{array}{l}\text { REF } \\
0.76(0.61-0.94)\end{array}$ & 0.014 \\
\hline N3 & $\begin{array}{l}\text { noAC } \\
\text { AC }\end{array}$ & $\begin{array}{l}21.0(16.6-24.3) \\
26.1(21.4-31.5)\end{array}$ & $\begin{array}{l}\text { REF } \\
0.64(0.46-0.90)\end{array}$ & 0.011 \\
\hline \multicolumn{5}{|c|}{ Stratified by margin status in matched cohort } \\
\hline R0 & $\begin{array}{l}\text { noAC } \\
\mathrm{AC}\end{array}$ & $\begin{array}{l}42.3(38.3-45.5) \\
49.2(43.8-54.4)\end{array}$ & $\begin{array}{l}\text { REF } \\
0.85(0.77-0.94)\end{array}$ & 0.001 \\
\hline $\mathrm{R} 1$ & $\begin{array}{l}\text { noAC } \\
\mathrm{AC}\end{array}$ & $\begin{array}{l}17.5(14.5-23.9) \\
22.3(19.7-28.1)\end{array}$ & $\begin{array}{l}\text { REF } \\
0.61(0.39-0.95)\end{array}$ & 0.028 \\
\hline \multicolumn{5}{|c|}{ Stratified by adjuvant radiotherapy status in matched cohort } \\
\hline $\begin{array}{l}\text { No adjuvant } \\
\text { radiotherapy }\end{array}$ & $\begin{array}{l}\text { noAC } \\
\mathrm{AC}\end{array}$ & $\begin{array}{l}42.0(37.1-46.2) \\
44.8(41.9-52.3)\end{array}$ & $\begin{array}{l}\text { REF } \\
0.86(0.78-0.96)\end{array}$ & 0.008 \\
\hline Adjuvant radiotherapy & $\begin{array}{l}\text { noAC } \\
\mathrm{AC}\end{array}$ & $\begin{array}{l}36.8(30.1-40.9) \\
51.1(40.2-67)\end{array}$ & $\begin{array}{l}\text { REF } \\
0.68(0.55-0.84)\end{array}$ & $<0.001$ \\
\hline
\end{tabular}

$A C$ adjuvant chemotherapy, $C I$ confidence interval, $I Q R$ interquartile range, noAC no adjuvant chemotherapy, $R E F$ referent 
Table 3 Multivariable cox regression model of survival of patients with resected ampullary adenocarcinoma in the matched cohort

\begin{tabular}{|c|c|c|c|}
\hline & & Hazard ratio $\left(\mathrm{CI}_{95 \%}\right)$ & $p$ value \\
\hline \multicolumn{4}{|l|}{ Hospital factors } \\
\hline \multirow[t]{5}{*}{ Center volume } & 1 (lowest) & REF & $<0.001$ \\
\hline & 2 & $0.84(0.71-0.98)$ & \\
\hline & 3 & $0.94(0.79-1.11)$ & \\
\hline & 4 & $0.88(0.74-1.04)$ & \\
\hline & 5 (highest) & $0.82(0.68-0.98)$ & \\
\hline \multirow[t]{3}{*}{ Facility type } & Community & REF & 0.8 \\
\hline & Academic & $0.97(0.85-1.10)$ & \\
\hline & Others & $1.11(0.95-1.28)$ & \\
\hline \multirow[t]{4}{*}{ Facility location } & Northeast & REF & $<0.001$ \\
\hline & South & $0.83(0.72-0.96)$ & \\
\hline & Midwest & $1.05(0.93-1.20)$ & \\
\hline & West & $1.07(0.92-1.24)$ & \\
\hline \multicolumn{4}{|l|}{ Patient factors } \\
\hline \multirow[t]{5}{*}{ Year of diagnosis } & 2006-2007 & REF & $<0.001$ \\
\hline & 2008-2009 & $1.01(0.86-1.19)$ & \\
\hline & 2010-2011 & $0.71(0.60-0.86)$ & \\
\hline & $2012-2013$ & $0.65(0.55-0.78)$ & \\
\hline & 2014-2016 & $0.68(0.56-0.83)$ & \\
\hline \multirow[t]{5}{*}{ Age at diagnosis (years) } & $36-50$ & REF & $<0.001$ \\
\hline & $51-65$ & $1.48(1.22-1.79)$ & \\
\hline & $66-80$ & $1.66(1.34-2.05)$ & \\
\hline & $\geq 80$ & $2.54(1.95-3.29)$ & \\
\hline & Missing & $0.94(0.23-3.88)$ & \\
\hline \multirow[t]{2}{*}{ Sex } & Male & REF & $<0.001$ \\
\hline & Female & $1.12(1.02-1.23)$ & \\
\hline \multirow[t]{2}{*}{ CDCC score } & $0-1$ & REF & $<0.001$ \\
\hline & $\geq 2$ & $1.16(0.97-1.40)$ & \\
\hline \multirow[t]{4}{*}{ Insurance status } & Uninsured & REF & $<0.001$ \\
\hline & Private insurance & $0.79(0.62-1.00)$ & \\
\hline & Medicaid & $0.73(0.58-0.91)$ & \\
\hline & Medicare & $0.90(0.68-1.18)$ & \\
\hline \multirow[t]{4}{*}{ Education level } & $\geq 21 \%$ & REF & 0.02 \\
\hline & $13-20.9 \%$ & $0.83(0.69-1.01)$ & \\
\hline & $7-12.9 \%$ & $0.95(0.81-1.12)$ & \\
\hline & $<7 \%$ & $1.03(0.90-1.18)$ & \\
\hline \multirow[t]{3}{*}{ Median income } & $\leq \$ 47,999$ & REF & $<0.001$ \\
\hline & $\$ 48,000-\$ 62,999$ & $0.83(0.73-0.95)$ & \\
\hline & $\geq \$ 63,000$ & $0.84(0.72-0.98)$ & \\
\hline \multicolumn{4}{|l|}{ Tumor factors } \\
\hline \multirow[t]{4}{*}{ Tumor grade } & Well & REF & $<0.001$ \\
\hline & Moderate & $0.84(0.69-1.03)$ & \\
\hline & Poor & $0.98(0.80-1.21)$ & \\
\hline & Anaplastic & $0.67(0.52-0.87)$ & \\
\hline \multirow[t]{4}{*}{ AJCC pathological T classification } & $\mathrm{T} 1$ & REF & $<0.001$ \\
\hline & $\mathrm{T} 2$ & $0.95(0.77-1.17)$ & \\
\hline & $\mathrm{T} 3$ & $1.83(1.51-2.23)$ & \\
\hline & $\mathrm{T} 4$ & $1.72(1.40-2.10)$ & \\
\hline \multirow[t]{4}{*}{ AJCC pathological N stage } & No & REF & $<0.001$ \\
\hline & N1 & $1.61(1.43-1.81)$ & \\
\hline & $\mathrm{N} 2$ & $2.34(2.01-2.71)$ & \\
\hline & N3 & $2.31(1.92-2.78)$ & \\
\hline Margin status & Negative & REF & $<0.001$ \\
\hline & Positive & $1.65(1.37-2.00)$ & \\
\hline Lymphovascular invasion & Absent & REF & 0.073 \\
\hline & Present & $1.35(1.18-1.55)$ & \\
\hline Treatment factors & & & \\
\hline Adjuvant radiotherapy & No & REF & $<0.001$ \\
\hline & Yes & $0.87(0.75-1.01)$ & \\
\hline Adjuvant chemotherapy & No & REF & $<0.001$ \\
\hline & Yes & $0.83(0.76-0.91)$ & \\
\hline
\end{tabular}

Additional variables included into the propensity matching omitted from tables were hospital factors (hospital distance), patient factors (race, residence), and tumor factors (lymph nodes examined)

$A J C C$ American Joint Commission on Cancer, $C D C C$ Charlson-Deyo comorbidity, $C I$ confidence interval, $R E F$ referent 
a

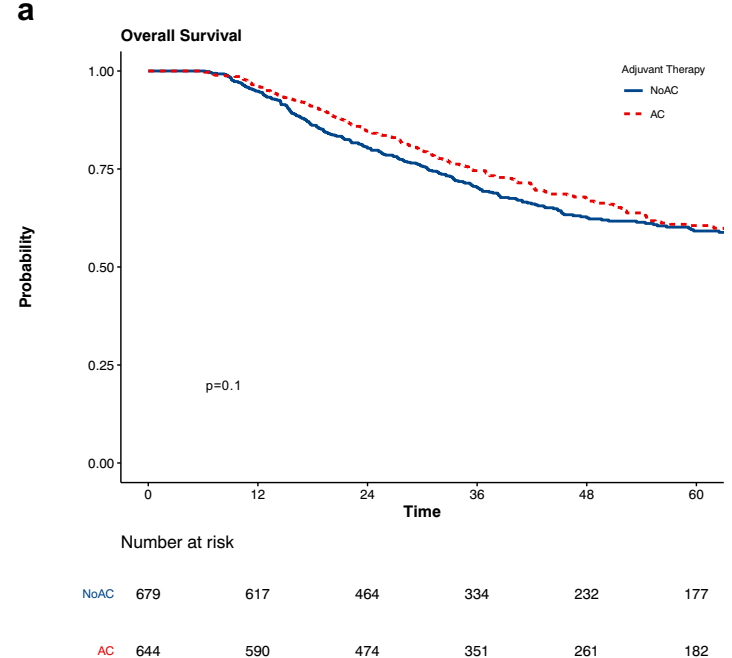

C

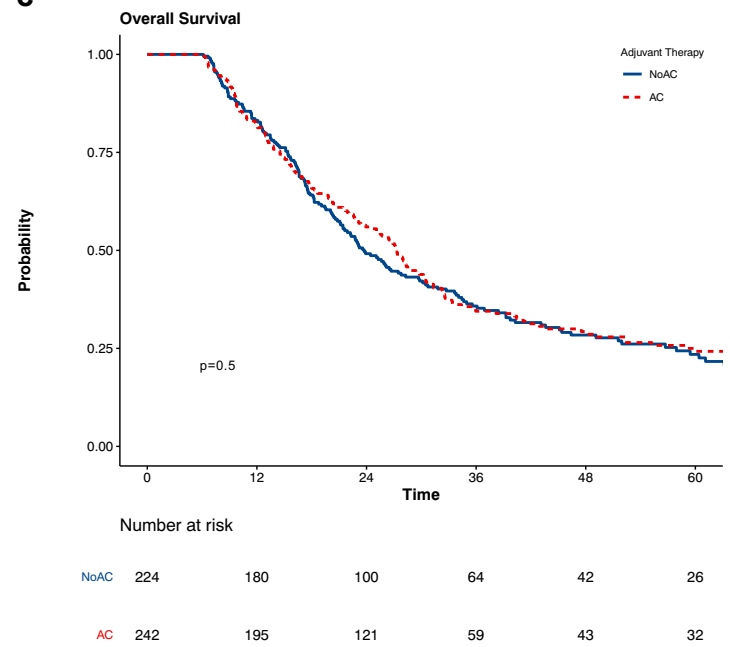

b

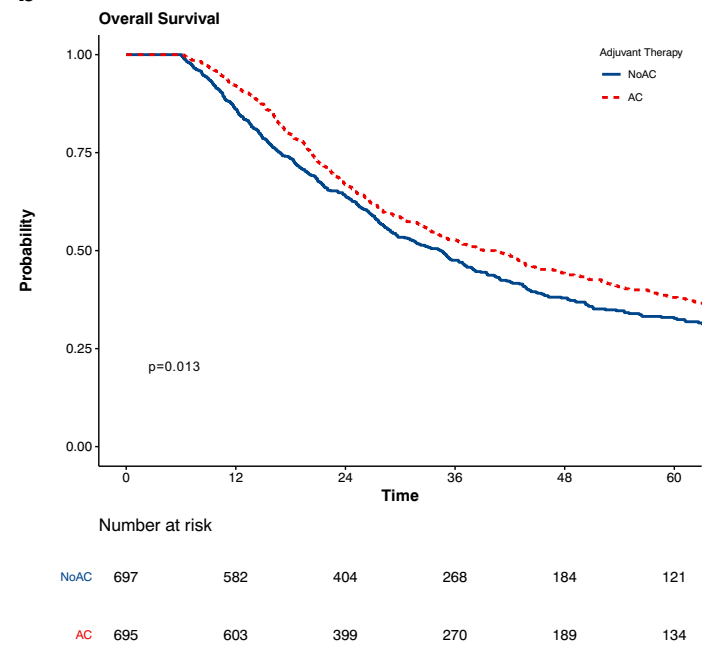

d

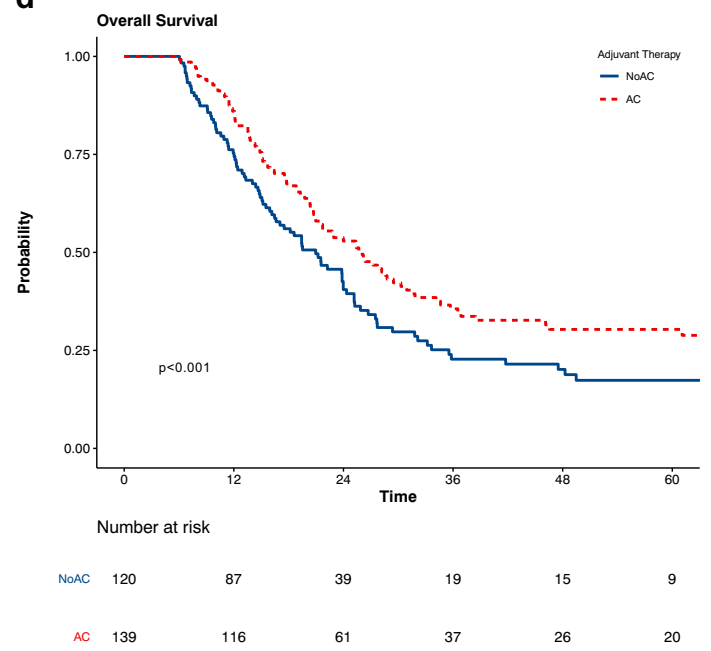

Fig. 2 Overall survival of adjuvant chemotherapy following resection for ampullary adenocarcinoma stratified by nodal status in matched cohorts: a N0, b N1, c N2, d N3

\section{Discussion}

Ampullary adenocarcinoma remains a relatively uncommon malignancy without broadly accepted protocols for optimal multimodality management following curative-intent resection. As such, there remains an ongoing dilemma regarding the role of AC after PD for ampullary adenocarcinoma, and practice varies significantly. In this large national registry analysis including 8307 patients, AC after resected ampullary adenocarcinoma was associated with improved survival after multivariable adjustment and accounting for treatment selection bias. Stratified analyses revealed that this benefit was maintained irrespective of pathological nodal involvement and resection margin status. Sensitivity landmark analyses excluding early postoperative deaths also demonstrated consistent findings favoring AC. ${ }^{34}$ As such, these data suggest a benefit to routine use of $\mathrm{AC}$ for ampullary adenocarcinoma, even in the absence of nodal involvement or compromised surgical margins. Broad acceptance of the routine use of AC for ampullary adenocarcinoma should be considered in the multimodality treatment of ampullary adenocarcinoma, just as in pancreatic cancer.

Current evidence for AC in resected ampullary adenocarcinoma is limited to retrospective case series. Recent institutional series by Ecker et al. ${ }^{26}$ ( $n=357$ patients; HR: 0.90 ; CI $_{95 \%}$ : 0.51-1.56), Bolm et al. ${ }^{30}$ with 214 patients (median: 85.0 vs 65.0 months), and Moekotte et al. ${ }^{31}$ with 1,163 patients (median: not reached vs 32 months) demonstrated no survival benefit with AC. However, these studies are limited by small institutional cohorts and selection bias. Subgroup analyses of the landmark ESPAC- $3^{20}$ RCT in patients with ampullary adenocarcinoma ( $n=297$ patients) demonstrated no statistically significant differences in survival between patients receiving gemcitabine, 5-fluorouracil, and no 
Table 4 Multivariable cox regression model of survival of patients with resected ampullary adenocarcinoma in matched cohort, with interactions between chemotherapy and nodal status and margin status

\begin{tabular}{llll}
\hline & & Hazard ratio $\left(\mathrm{CI}_{95 \%}\right)$ & $p$ value \\
\hline Interaction by nodal status & & & \\
$\begin{array}{l}\text { Adjuvant chemotherapy } \times \text { AJCC } \\
\text { pathological N stage }\end{array}$ & $\mathrm{N} 0+$ noAC & REF & 0.001 \\
& $\mathrm{~N} 0+\mathrm{AC}$ & $0.81(0.68-0.97)$ & \\
& $\mathrm{N} 1+\mathrm{noAC}$ & $1.60(1.37-1.88)$ & \\
& $\mathrm{N} 1+\mathrm{AC}$ & $0.65(0.61-0.70)$ & \\
& $\mathrm{N} 2+\mathrm{noAC}$ & $2.15(1.75-2.64)$ & \\
& $\mathrm{N} 2+\mathrm{AC}$ & $0.73(0.59-0.90)$ & \\
& $\mathrm{N} 3+\mathrm{noAC}$ & $2.51(1.95-3.24)$ & \\
& $\mathrm{N} 3+\mathrm{AC}$ & $0.59(0.44-0.78)$ & \\
Interaction by margin status & & & \\
Adjuvant chemotherapy $\times$ margin status & $\mathrm{R} 0+\mathrm{noAC}$ & $\mathrm{REF}$ & \\
& $\mathrm{R} 0+\mathrm{AC}$ & $0.85(0.77-0.94)$ & \\
& $\mathrm{R} 1+\mathrm{noAC}$ & $2.00(1.55-2.59)$ & \\
& $\mathrm{R} 1+\mathrm{AC}$ & $0.69(0.48-1.00)$ & \\
\hline
\end{tabular}

$A C$ adjuvant chemotherapy, $C D C C$ Charlson-Deyo comorbidity, $C I$ confidence interval, noAC no adjuvant chemotherapy, $R E F$ referent chemotherapy (median: 70.8 vs 57.8 vs 40.6 months). This is possibly a result of a type II error. The only level I evidence on the role of $\mathrm{AC}$ in clinical practice is drawn from subgroup analyses of RCTs ${ }^{18,20,21}$ in periampullary cancers, which have their own limitations. This large study, while still retrospective, used robust methods to account for treatment selection bias and still demonstrated survival benefit with AC.

The presence of high-risk factors, such as nodal involvement or positive margins, is commonly used to select patients for adjuvant therapy, as evidence by the distribution AC use in the unmatched cohort. To the authors' knowledge, no published studies have explored the role of AC specifically in patients with node-negative disease or negative margins.
Such treatment decisions likely reflect an estimation of the risk of systemic recurrence, which clearly is lower in patients with node-negative, margin-negative resections. However, systemic recurrence in such patients may still be as high as $40 \%{ }^{8,37}$, and local recurrence as high as $50 \%{ }^{38,39}$ Our results suggest that $\mathrm{AC}$ has a role in these subgroups of patients by reducing or delaying recurrence and prolonging survival. Nevertheless, there may be a more select subgroup of patients in whom the benefit of AC does not outweigh the risk ${ }^{40}$, especially those with intestinal-type rather than pancreaticobiliary-type tumors, given their more favorable overall prognosis. ${ }^{41}$ Unfortunately, NCDB data do not allow these subtypes to be distinguished. However, if intestinal-type
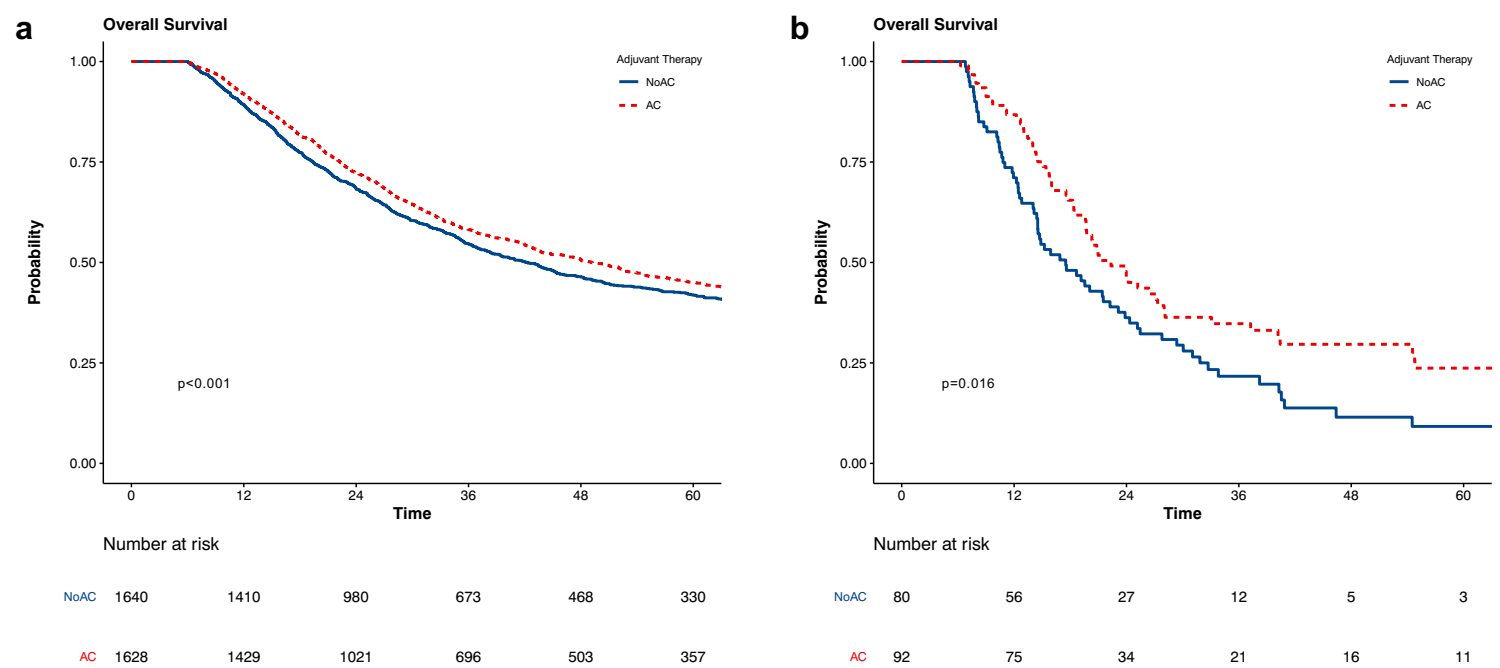

Fig. 3 Overall survival of adjuvant chemotherapy following resection for ampullary adenocarcinoma stratified by margin status in matched cohorts: a R0, b R1 
tumors have no true benefit or less benefit from adjuvant chemotherapy, the implication from the survival data is that pancreaticobiliary-type tumors have even more benefit than estimated.

Several limitations of our study should be acknowledged. First, despite the use of PSM to address treatment selection bias, the potential for residual bias remains in this retrospective cohort study. Second, the duration of adjuvant chemotherapy and the specific regimens used are not available from NCDB. Third, this study did not assess the role of neoadjuvant RT, which may or may not be associated with a similar survival benefit. Fourth, pathologic assessment of tumors in the periampullary region can be challenging, as the site or origin (true ampullary vs other peri-ampullary) may be difficult to ascertain for larger tumors. However, this limitation applies to any study that uses histopathologic analysis for diagnosis. Fifth, patients with survival of $<6$ months were excluded as it is likely that these patient cohorts may not have completed course of adjuvant chemotherapy due to death. However, it is unclear if these patients had complications related to adjuvant therapy. Finally, because NCDB does not include data on recurrence patterns or disease-free survival, we can only speculate as to whether improved survival was associated with local or systemic disease control.

\section{Conclusion}

In this large nationwide retrospective study, AC was associated with a survival benefit in patients with resected ampullary adenocarcinoma, regardless of pathological nodal involvement, resection margin status, and receipt of adjuvant radiotherapy. These data suggest AC should be broadly considered in the multimodality treatment of ampullary adenocarcinoma.

Supplementary Information The online version contains supplementary material available at https://doi.org/10.1007/s11605-020-04879-x.

\section{Compliance with Ethical Standards}

Conflict of Interest The authors declare that they have no conflict of interest.

Open Access This article is licensed under a Creative Commons Attribution 4.0 International License, which permits use, sharing, adaptation, distribution and reproduction in any medium or format, as long as you give appropriate credit to the original author(s) and the source, provide a link to the Creative Commons licence, and indicate if changes were made. The images or other third party material in this article are included in the article's Creative Commons licence, unless indicated otherwise in a credit line to the material. If material is not included in the article's Creative Commons licence and your intended use is not permitted by statutory regulation or exceeds the permitted use, you will need to obtain permission directly from the copyright holder. To view a copy of this licence, visit http://creativecommons.org/licenses/by/4.0/.

\section{References}

1. Kim K, Chie EK, Jang JY, et al. Role of adjuvant chemoradiotherapy for ampulla of Vater cancer. Int J Radiat Oncol Biol Phys. 2009;75(2):436-441.

2. Narang AK, Miller RC, Hsu CC, et al. Evaluation of adjuvant chemoradiation therapy for ampullary adenocarcinoma: the Johns Hopkins Hospital-Mayo Clinic collaborative study. Radiat Oncol. 2011;6:126.

3. Brown KM, Tompkins AJ, Yong S, Aranha GV, Shoup M. Pancreaticoduodenectomy is curative in the majority of patients with node-negative ampullary cancer. Arch Surg. 2005;140(6): 529-532; discussion 532-523.

4. Kamarajah SK. Pancreaticoduodenectomy for periampullary tumours: a review article based on Surveillance, End Results and Epidemiology (SEER) database. Clin Transl Oncol. 2018;20(9): $1153-1160$

5. Cameron JL, He J. Two thousand consecutive pancreaticoduodenectomies. $J$ Am Coll Surg. 2015;220(4):530 536.

6. Stiles ZE, Behrman SW, Deneve JL, et al. Ampullary adenocarcinoma: Defining predictors of survival and the impact of adjuvant therapy following surgical resection for stage I disease. J Surg Oncol. 2018;117(7):1500-1508.

7. O'Connell JB, Maggard MA, Manunga J, Jr., et al. Survival after resection of ampullary carcinoma: a national population-based study. Ann Surg Oncol. 2008;15(7):1820-1827.

8. Kim RD, Kundhal PS, McGilvray ID, et al. Predictors of failure after pancreaticoduodenectomy for ampullary carcinoma. $J \mathrm{Am}$ Coll Surg. 2006;202(1):112-119.

9. Todoroki T, Koike N, Morishita Y, et al. Patterns and predictors of failure after curative resections of carcinoma of the ampulla of Vater. Ann Surg Oncol. 2003;10(10):1176-1183.

10. Sikora SS, Balachandran P, Dimri K, et al. Adjuvant chemoradiotherapy in ampullary cancers. Eur J Surg Oncol. 2005;31(2): 158-163.

11. Hsu HP, Yang TM, Hsieh YH, Shan YS, Lin PW. Predictors for patterns of failure after pancreaticoduodenectomy in ampullary cancer. Ann Surg Oncol. 2007;14(1):50-60.

12. Oettle H, Neuhaus P, Hochhaus A, et al. Adjuvant chemotherapy with gemcitabine and long-term outcomes among patients with resected pancreatic cancer: the CONKO-001 randomized trial. JAMA. 2013;310(14):1473-1481.

13. Oettle H, Post S, Neuhaus P, et al. Adjuvant chemotherapy with gemcitabine vs observation in patients undergoing curative-intent resection of pancreatic cancer: a randomized controlled trial. JAMA. 2007;297(3):267-277.

14. Neoptolemos JP, Dunn JA, Stocken DD, et al. Adjuvant chemoradiotherapy and chemotherapy in resectable pancreatic cancer: a randomised controlled trial. Lancet. 2001;358(9293):1576-1585.

15. Neoptolemos JP, Palmer DH, Ghaneh P, et al. Comparison of adjuvant gemcitabine and capecitabine with gemcitabine monotherapy in patients with resected pancreatic cancer (ESPAC-4): a multicentre, open-label, randomised, phase 3 trial. Lancet. 2017;389(10073):1011-1024.

16. Conroy T, Hammel P, Hebbar M, et al. FOLFIRINOX or Gemcitabine as Adjuvant Therapy for Pancreatic Cancer. N Engl J Med. 2018;379(25):2395-2406.

17. Neoptolemos JP, Stocken DD, Friess H, et al. A randomized trial of chemoradiotherapy and chemotherapy after resection of pancreatic cancer. N Engl J Med. 2004;350(12):1200-1210.

18. Morak MJM, van der Gaast A, Incrocci L, et al. Adjuvant IntraArterial Chemotherapy and Radiotherapy Versus Surgery Alone in Resectable Pancreatic and Periampullary Cancer. Annals of Surgery. 2008;248(6):1031-1041. 
19. Klinkenbij1 JH, Jeekel J, Sahmoud T, et al. Adjuvant radiotherapy and 5-fluorouracil after curative resection of cancer of the pancreas and periampullary region: phase III trial of the EORTC gastrointestinal tract cancer cooperative group. Ann Surg. 1999;230(6):776782; discussion 782-774.

20. Neoptolemos JP, Moore MJ, Cox TF, et al. Effect of adjuvant chemotherapy with fluorouracil plus folinic acid or gemcitabine vs observation on survival in patients with resected periampullary adenocarcinoma: the ESPAC-3 periampullary cancer randomized trial. Jama. 2012;308(2):147-156.

21. Smeenk HG, van Eijck CH, Hop WC, et al. Long-term survival and metastatic pattern of pancreatic and periampullary cancer after adjuvant chemoradiation or observation: long-term results of EORTC trial 40891. Ann Surg. 2007;246(5):734-740.

22. Acharya A, Markar SR, Sodergren MH, et al. Meta-analysis of adjuvant therapy following curative surgery for periampullary adenocarcinoma. Br J Surg. 2017;104(7):814-822.

23. Horgan AM, Amir E, Walter T, Knox JJ. Adjuvant therapy in the treatment of biliary tract cancer: a systematic review and meta-analysis. J Clin Oncol. 2012;30(16):1934-1940.

24. Sandhu V, Wedge DC, Bowitz Lothe IM, et al. The Genomic Landscape of Pancreatic and Periampullary Adenocarcinoma. Cancer Res. 2016;76(17):5092-5102.

25. Schiergens TS, Reu S, Neumann J, et al. Histomorphologic and molecular phenotypes predict gemcitabine response and overall survival in adenocarcinoma of the ampulla of Vater. Surgery. 2015;158(1):151-161.

26. Ecker BL, Vollmer CM, Jr., Behrman SW, et al. Role of Adjuvant Multimodality Therapy After Curative-Intent Resection of Ampullary Carcinoma. JAMA Surg. 2019.

27. Krishnan S, Rana V, Evans DB, et al. Role of adjuvant chemoradiation therapy in adenocarcinomas of the ampulla of vater. Int $J$ Radiat Oncol Biol Phys. 2008;70(3):735-743.

28. Lazaryan A, Kalmadi S, Almhanna K, Pelley R, Kim R. Predictors of clinical outcomes of resected ampullary adenocarcinoma: a single-institution experience. Eur J Surg Oncol. 2011;37(9):791797.

29. Zhou J, Hsu CC, Winter JM, et al. Adjuvant chemoradiation versus surgery alone for adenocarcinoma of the ampulla of Vater. Radiother Oncol. 2009;92(2):244-248.

30. Bolm L, Ohrner K, Nappo G, et al. Adjuvant therapy is associated with improved overall survival in patients with pancreatobiliary or mixed subtype ampullary cancer after pancreatoduodenectomy - A multicenter cohort study. Pancreatology. 2020.
31. Moekotte AL, Malleo G, van Roessel S, et al. Gemcitabine-based adjuvant chemotherapy in subtypes of ampullary adenocarcinoma: international propensity score-matched cohort study. Br J Surg. 2020.

32. Bilimoria KY, Bentrem DJ, Ko CY, et al. Validation of the 6th edition AJCC Pancreatic Cancer Staging System: report from the National Cancer Database. Cancer. 2007;110(4):738-744.

33. Merkow RP, Rademaker AW, Bilimoria KY. Practical Guide to Surgical Data Sets: National Cancer Database (NCDB). JAMA Surg. 2018.

34. Giobbie-Hurder A, Gelber RD, Regan MM. Challenges of guarantee-time bias. J Clin Oncol. 2013;31(23):2963-2969.

35. Austin PC. The use of propensity score methods with survival or time-to-event outcomes: reporting measures of effect similar to those used in randomized experiments. Stat Med. 2014;33(7): $1242-1258$

36. Kamarajah SK, Sonnenday CJ, Cho CS, et al. Association of Adjuvant Radiotherapy With Survival After Margin-negative Resection of Pancreatic Ductal Adenocarcinoma: A Propensitymatched National Cancer Database (NCDB) Analysis. Ann Surg. 2019.

37. Lee JH, Whittington R, Williams NN, et al. Outcome of pancreaticoduodenectomy and impact of adjuvant therapy for ampullary carcinomas. Int J Radiat Oncol Biol Phys. 2000;47(4):945953.

38. Junrungsee S, Kittivarakul E, Ko-iam W, Lapisatepun W, Sandhu T, Chotirosniramit A. Prognostic Factors and Survival of Patients with Carcinoma of the Ampulla of Vater after Pancreaticoduodenectomy. Asian Pac J Cancer Prev. 2017;18(1):225-229.

39. de Castro SM, Kuhlmann KF, van Heek NT, et al. Recurrent disease after microscopically radical (R0) resection of periampullary adenocarcinoma in patients without adjuvant therapy. $J$ Gastrointest Surg. 2004;8(7):775-784; discussion 784.

40. Moekotte AL, Lof S, Van Roessel S, et al. Histopathologic Predictors of Survival and Recurrence in Resected Ampullary Adenocarcinoma: International Multicenter Cohort Study. Ann Surg. 2019.

41. Chang DK, Jamieson NB, Johns AL, et al. Histomolecular phenotypes and outcome in adenocarcinoma of the ampulla of vater. $J$ Clin Oncol. 2013;31(10):1348-1356.

Publisher's Note Springer Nature remains neutral with regard to jurisdictional claims in published maps and institutional affiliations. 